MATHEMATICS OF COMPUTATION

Volume 71, Number 238 , Pages 683-701

S 0025-5718(01)01337-0

Article electronically published on October 4, 2001

\title{
SZEGÖ QUADRATURE FORMULAS FOR CERTAIN JACOBI-TYPE WEIGHT FUNCTIONS
}

\author{
LEYLA DARUIS, PABLO GONZÁLEZ-VERA, AND OLAV NJÅSTAD
}

\begin{abstract}
In this paper we are concerned with the estimation of integrals on the unit circle of the form $\int_{0}^{2 \pi} f\left(e^{i \theta}\right) \omega(\theta) d \theta$ by means of the so-called Szegö quadrature formulas, i.e., formulas of the type $\sum_{j=1}^{n} \lambda_{j} f\left(x_{j}\right)$ with distinct nodes on the unit circle, exactly integrating Laurent polynomials in subspaces of dimension as high as possible. When considering certain weight functions $\omega(\theta)$ related to the Jacobi functions for the interval $[-1,1]$, nodes $\left\{x_{j}\right\}_{j=1}^{n}$ and weights $\left\{\lambda_{j}\right\}_{j=1}^{n}$ in Szegö quadrature formulas are explicitly deduced. Illustrative numerical examples are also given.
\end{abstract}

\section{INTRODUCTION}

When considering the approximate calculation of integrals of the form

$$
I_{\psi}(f)=\int_{a}^{b} f(x) d \psi(x), \quad(-\infty \leq a<b \leq+\infty)
$$

by means of a quadrature formula like

$$
I_{n}(f)=\sum_{j=1}^{n} A_{j} f\left(x_{j}\right)
$$

it is well known that distinct nodes $\left\{x_{j}\right\}_{j=1}^{n}$ in $(a, b)$ and positive weights $\left\{A_{j}\right\}_{j=1}^{n}$ can be uniquely determined so that $I_{\psi}(p)=I_{n}(p)$ for any $p$ in $\Pi_{2 n-1}$ (the space of polynomials of degree $2 n-1$ at most). In this case, $I_{n}(f)$ represents the $n$th Gauss-Christoffel quadrature formula so that its nodes $\left\{x_{j}\right\}_{j=1}^{n}$ are the zeros of the $n$th orthogonal polynomial with respect to $d \psi$.

In this paper, we shall be concerned with the estimation of integrals on the unit circle i.e.,

$$
I_{\psi}(f)=\int_{0}^{2 \pi} f\left(e^{i \theta}\right) d \psi(\theta)
$$

Received by the editor February 11, 2000 and, in revised form, July 10, 2000.

2000 Mathematics Subject Classification. Primary 41A55, 33C45.

Key words and phrases. Weight functions, quadrature formulas, orthogonal polynomials, Szegö polynomials, error bounds.

The work of the first author was performed as part of a grant of the Gobierno de Canarias.

The work of the second author was supported by the Scientific Research Project of the Spanish D.G.E.S. under contract PB96-1029. 
where $\psi$ is a positive Borel measure on $[0,2 \pi]$. For our purpose, instead of a measure $\psi$, we could deal with a distribution function on $[0,2 \pi]$ i.e., a real valued nondecreasing function with infinitely many points of increase on $[0,2 \pi]$, (see [7]). In order to make the paper consistent throughout, measures will be used.

We shall make use of the so-called Szegö quadrature formulas which are to some extent analogous on the unit circle to Gauss quadrature formulas on the real line. The Szegö quadrature formulas were introduced by Jones, Njåstad and Thron in [6] in connection with the trigonometric moment problem, where orthogonal polynomials on the unit circle with respect to $\psi$ (Szegö polynomials), become crucial. Except for very special measures $\psi$, it is difficult to obtain explicit expressions for these polynomials. For instance, it is known that for the Lebesgue measure, the sequence of monic Szegö polynomials is given by $\rho_{n}(z)=z^{n}$ for all $n$. If we consider the measure

$$
d \psi(\theta)=\frac{d \theta}{2 \pi\left|h\left(e^{i \theta}\right)\right|^{2}},
$$

where $h(z)=\prod_{i=1}^{k}\left(z-\alpha_{i}\right)$ with $\left|\alpha_{i}\right|<1$, then, (1.1) represents a rational modification of the Lebesgue measure and the system of Szegö polynomials is given explicitly in 11, pp. 289-290. For further details see [5].

Our main interest is centered on calculating explicit expressions for Szegö polynomials for certain Jacobi-type weight functions, which allows us to obtain an explicit formula for Szegö quadrature formulas.

We shall use the notation $\mathbb{T}=\{z:|z|=1\}$ and $\mathbb{D}=\{z:|z|<1\}$ for the unit circle and the open unit disc, respectively. Also, for $p$ and $q$ nonnegative integers, $p \leq q, \Lambda_{p, q}$ will denote the space of Laurent polynomials of the form $L(z)=\sum_{j=p}^{q} \alpha_{j} z^{j}, \alpha_{j} \in \mathbb{C}$. $\Lambda$ will denote the space of all Laurent polynomials and $\Pi$ the space of algebraic polynomials.

The paper is organized as follows. In Section 2, preliminary results concerning Szegö polynomials, associated polynomials, Szegö quadrature formulas and second kind measures are given. In Sections 3 we obtain explicit representations for Szegö quadrature formulas with respect to the measures $d \psi(\theta)=\frac{\sin ^{2}(\theta)}{2 \pi} d \theta$, $d \psi(\theta)=\frac{1+\cos \theta}{2 \pi} d \theta$ and $d \psi(\theta)=\frac{1-\cos \theta}{2 \pi} d \theta$, respectively, along with integral error expressions and computable error bounds when considering analytic integrands. Finally, in Section 4, illustrative numerical examples are given. For other results concerning Szegö quadrature formulas see [3].

\section{Preliminary Results}

Szegö polynomials. Let $\psi$ be a positive Borel measure on $[0,2 \pi]$. Let us consider the following inner product in the linear space $\Pi$ of polynomials with complex coefficients:

$$
(f, g)_{\psi}=\int_{0}^{2 \pi} f\left(e^{i \theta}\right) \overline{g\left(e^{i \theta}\right)} d \psi(\theta) .
$$

By applying the Gram-Schmidt orthogonalization process to $\left\{1, z, \ldots, z^{n}\right\}$, an orthogonal basis $\left\{\rho_{k}(z)\right\}_{k=0}^{n}$ can be deduced such that $\operatorname{deg}\left(\rho_{k}\right)=k$ and

$$
\left(\rho_{j}, \rho_{k}\right)_{\psi}=K_{j} \delta_{j, k}, \quad K_{j}>0,0 \leq j, k \leq n .
$$

Thus, when taking $\rho_{n}(z)$ monic for each $n$, the so-called monic orthogonal polynomials on the unit circle $\mathbb{T}$ or Szegö polynomials are obtained. These polynomials 
satisfy recurrence relations

$$
\begin{aligned}
& \rho_{0}(z)=\rho_{0}^{*}(z)=1, \\
& \rho_{n}(z)=z \rho_{n-1}(z)+\delta_{n} \rho_{n-1}^{*}(z), \quad n=1,2,3, \ldots, \\
& \rho_{n}^{*}(z)=\bar{\delta}_{n} z \rho_{n-1}(z)+\rho_{n-1}^{*}(z), \quad n=1,2,3, \ldots,
\end{aligned}
$$

where $\delta_{n}:=\rho_{n}(0), n=1,2,3, \ldots$, are called the reflection coefficients and $\rho_{n}^{*}(z)=$ $z^{n} \overline{\rho_{n}(1 / \bar{z})}$. Conversely, if a sequence $\left\{\rho_{n}\right\}$ such that, for each $n$ the polynomial $\rho_{n}$ has exact degree $n$ and satisfies (2.1), is given, then there exists a measure $\psi$ so that $\left\{\rho_{n}\right\}$ is the corresponding sequence of monic Szegö polynomials.

In general, it is difficult to obtain an explicit expression for these polynomials. If we want to calculate them, we can make use of the so-called Levinson algorithm (see e.g., [2]).

Associated Szegö polynomials. Let $\mu_{k}=\int_{0}^{2 \pi} e^{-k i \theta} d \psi(\theta), k \in \mathbb{Z}$, then $\left\{\mu_{k}\right\}_{k}$ is called the moment sequence with respect to $\psi$. Note that $\mu_{-k}=\overline{\mu_{k}}$. Let $\left\{\pi_{n}\right\}_{n}$ be a sequence of polynomials defined in terms of the sequence $\left\{\rho_{n}\right\}_{n}$ and the moments $\left\{\mu_{k}\right\}$, by means of

$$
\pi_{n}(z):=\left\{\begin{array}{lll}
\int_{0}^{2 \pi} \frac{z+e^{i \theta}}{z-e^{i \theta}}\left(\rho_{n}\left(e^{i \theta}\right)-\rho_{n}(z)\right) d \psi(\theta) & \text { if } & n=1,2,3, \ldots, \\
-\mu_{0} & \text { if } & n=0 .
\end{array}\right.
$$

These polynomials are called the associated Szegö polynomials and they satisfy the relations

$$
\begin{aligned}
& \pi_{0}(z):=-\mu_{0}, \\
& z \pi_{n-1}(z)-\delta_{n} \pi_{n-1}^{*}(z)=\pi_{n}(z), n=1,2, \ldots, \\
& \delta_{n} z \pi_{n-1}(z)-\pi_{n-1}^{*}(z)=-\pi_{n}^{*}(z), n=1,2, \ldots,
\end{aligned}
$$

where $\delta_{n}$ are the reflection coefficients and $\pi_{n}^{*}(z)=z^{n} \overline{\pi(1 / \bar{z})}$.

Szegö quadrature formulas. We are concerned with the estimation of integrals on the unit circle $\mathbb{T}=\{z:|z|=1\}$ of the form

$$
I_{\psi}(f)=\int_{0}^{2 \pi} f\left(e^{i \theta}\right) d \psi(\theta)
$$

by a quadrature formula

$$
I_{n}(f)=\sum_{j=1}^{n} \lambda_{j} f\left(x_{j}\right)
$$

where the parameters $\lambda_{j}$ and $x_{j}, 1 \leq j \leq n$, are determined so that $I_{\psi}(f)=I_{n}(f)$ for all $f \in \Lambda_{-(n-1), n-1}$. Furthermore, the nodes $\left\{x_{j}\right\}_{j=1}^{n}$ should lie on the unit circle. We know that the zeros of $\rho_{n}$ lie in $\mathbb{D}$ (see [1], p. 184). In order to construct a polynomial with zeros on $\mathbb{T}$, we define

$$
B_{n}\left(z, \tau_{n}\right)=\rho_{n}(z)+\tau_{n} \rho_{n}^{*}(z), \quad\left|\tau_{n}\right|=1, \forall n .
$$

This polynomial has $n$ simple zeros on $\mathbb{T}$ (see [6]) and $\left\{B_{n}\left(z, \tau_{n}\right)\right\}_{n}$ is called a sequence of para-orthogonal polynomials with respect to $\psi$. If $x_{j}, 1 \leq j \leq n$, are the zeros of $B_{n}\left(z, \tau_{n}\right)$, then (2.4) is called the $n$-point Szegö quadrature formula.

Let us define

$$
A_{n}\left(z, \tau_{n}\right)=\pi_{n}(z)-\tau_{n} \pi_{n}^{*}(z),\left|\tau_{n}\right|=1, \forall n,
$$


where $\pi_{n}$ is the polynomial associated with $\rho_{n}$ with respect to $\psi$, then the weights $\lambda_{j}, 1 \leq j \leq n$, of the $n$-point Szegö quadrature formula, can be expressed as ([5])

$$
\lambda_{j}=\frac{-1}{2 x_{j}} \frac{A_{n}\left(x_{j}, \tau_{n}\right)}{B_{n}^{\prime}\left(x_{j}, \tau_{n}\right)}, 1 \leq j \leq n,
$$

where $x_{j}, 1 \leq j \leq n$, are the zeros of $B_{n}\left(z, \tau_{n}\right)$.

To the measure $\psi$, we can associate Carathéodory functions $F_{\psi}$ that is, functions analytic in $\mathbb{D}=\{z:|z|<1\}$ with $\Re\left(F_{\psi}\right) \geq 0$ for $z \in \mathbb{D}$. We can express $F_{\psi}$ as

$$
F_{\psi}(z)=\int_{0}^{2 \pi} \frac{e^{i \theta}+z}{e^{i \theta}-z} d \psi(\theta)+i c, \quad c \in \mathbb{R}
$$

which is known as the Herglotz-Riesz transform for the measure $\psi$. Clearly $c=$ $\Im F_{\psi}(0)$.

Conversely, given a Carathéodory function $F$, there exists a positive Borel measure $\psi$ on $[0,2 \pi]$ such that (2.8) holds.

Let us define the rational functions

$$
R_{n}\left(z, \tau_{n}\right)=\frac{A_{n}\left(z, \tau_{n}\right)}{B_{n}\left(z, \tau_{n}\right)},\left|\tau_{n}\right|=1, \forall n,
$$

where $B_{n}$ and $A_{n}$ are as in formulas (2.5) and (2.6), respectively. The role played by $F_{\psi}(z)$ and $R_{n}\left(z, \tau_{n}\right)$ with respect to the Szegö formula is displayed in the following

Lemma 2.1 (See [5]). Let $G$ be a neighbourdhood of $\mathbb{T}$ with boundary $\Gamma$ consisting of a finite number of rectifiable Jordan curves, and let $\frac{f(z)}{z}$ be analytic in $G$. Then, for each $n=1,2, \ldots$ it holds that

$$
E_{n}(f)=I_{\psi}(f)-I_{n}(f)=\frac{1}{2 \pi i} \int_{\Gamma}\left(F_{\psi}(z)-R_{n}\left(z, \tau_{n}\right)\right) g(z) d z,
$$

where $g(z)=-\frac{f(z)}{2 z}$ and the rational function $R_{n}\left(z, \tau_{n}\right)$ is given as in formula (2.9).

Remark 2.2. The Lemma in particular covers the situation where $G$ contains the whole of $\hat{\mathbb{C}}-\mathbb{D}$, and $\frac{f(z)}{z}$ is analytic at $z=\infty$. Furthermore, assuming that $\frac{f(z)}{z}$ is analytic in $G$ is not a restriction, since when $f(0) \neq 0$, we can deal with the function $f(z)-f(0)$.

So, it is easy to see that

$$
\left|E_{n}(f)\right| \leq \frac{1}{4 \pi} \max _{\xi \in \Gamma}\left\{\left|\frac{f(\xi)}{\xi}\right|\right\} \int_{\Gamma}\left|F_{\psi}(z)-R_{n}\left(z, \tau_{n}\right)\right| d z .
$$

Second kind measure. From the recurrence relations (2.1), and the Favard Theorem ([6]) one sees that the sequence $\left\{\pi_{n}\right\}$ of associated polynomials is also orthogonal with respect to a measure $\widetilde{\psi}$ which is said to be of the second kind associated with $\psi$. In order to calculate this measure, the following result is required,

Theorem 2.3 (9]). Let $F$ be analytic in $\mathbb{T}$ and suppose that $F$ has simple poles at $\left\{z_{k}\right\}_{k=1}^{n}$ with $\left|z_{k}\right|=1$ in such a way that the limits

$$
\lim _{z \rightarrow z_{k}}\left(z-z_{k}\right) F(z)=\gamma_{k}
$$

exist and $\bar{z}_{k} \gamma_{k} \in \mathbb{R}$. Assume that the nontangencial boundary values

$$
\lim _{z \rightarrow e^{i \theta}} \Re\left\{F(z)-\sum_{k=1}^{n} \frac{\gamma_{k}}{z-z_{k}}\right\}
$$


exist a.e. on $[0,2 \pi]$ and are $L_{p}$-integrable on $[0,2 \pi]$ with $p \in(1,+\infty)$. Then,

$$
F(z)=\int_{0}^{2 \pi} \frac{e^{i \theta}+z}{e^{i \theta}-z} d \psi(\theta)+i \Im(F(0))
$$

with

$$
d \psi(\theta)=(\Re(F(z))) d \theta-\sum_{k=1}^{n} \frac{2 \pi \gamma_{k}}{z_{k}} d \delta\left(e^{i \theta}-z_{k}\right),
$$

where $\Re\left(F\left(e^{i \theta}\right)\right)=\lim _{z \rightarrow e^{i \theta}} \Re(f(z))$ means the nontangential limit and where

$$
d \delta\left(e^{i \theta}-z_{k}\right)=\left\{\begin{array}{lll}
1 & \text { if } \quad e^{i \theta}=z_{k} \\
0 & \text { if } & e^{i \theta} \neq z_{k} .
\end{array}\right.
$$

It can also be shown (see [10]) that

Theorem 2.4. If $F$ is the Carathéodory function corresponding to the measure $\psi$ and $\widetilde{\psi}$ is the measure of the second kind associated with $\psi$, then, $G=\frac{1}{F}$ is the Carathéodory function corresponding to $\widetilde{\psi}$.

\section{Chebyshev Weight Functions}

Throughout the rest of the paper we will restrict ourselves to an absolutely continuous measure $\psi$, i.e., $d \psi(\theta)=\omega(\theta) d \theta, \omega(\theta)>0$ a.e. on $[0,2 \pi]$. Thus, instead of a measure $\psi$ we will deal with a weight function $\omega(\theta)$. A special case is the so-called Jacobi-type weight functions.

By a Jacobi-type weight function we mean a function of the form $\omega(\theta)=$ $h(\cos \theta)|\sin \theta|, \theta \in[0,2 \pi]$ where $h(x)=(1-x)^{\alpha}(1+x)^{\beta}, x \in[-1,1], \alpha, \beta>-1$.

Thus,

$$
\begin{aligned}
\omega(\theta) & =h(\cos \theta)|\sin \theta| \\
& =(1-\cos \theta)^{\alpha}(1+\cos \theta)^{\beta} \sqrt{1-\cos ^{2} \theta} \\
& =(1-\cos \theta)^{\alpha}(1+\cos \theta)^{\beta} \sqrt{(1-\cos \theta)(1+\cos \theta)} \\
& =(1-\cos \theta)^{\alpha+1 / 2}(1+\cos \theta)^{\beta+1 / 2} .
\end{aligned}
$$

Observe that we can write

$$
\omega(\theta)=2^{\gamma_{1}+\gamma_{2}}\left|e^{i \theta}-1\right|^{2 \gamma_{1}}\left|e^{i \theta}+1\right|^{2 \gamma_{2}}=2^{\gamma_{1}+\gamma_{2}}\left|\sin \frac{\theta}{2}\right|^{2 \gamma_{1}}\left|\cos \frac{\theta}{2}\right|^{2 \gamma_{2}}
$$

with $\gamma_{1}=\alpha+\frac{1}{2}$; and $\gamma_{2}=\beta+\frac{1}{2}$. Since $\alpha, \beta>-1$, then $\gamma_{1}, \gamma_{2}>-\frac{1}{2}$. In this paper we shall restrict ourselves to the following cases

$$
\begin{array}{lll}
\alpha=\frac{1}{2}, & \beta=\frac{1}{2}, & \omega(\theta)=\sin ^{2} \theta, \\
\alpha=-\frac{1}{2}, & \beta=\frac{1}{2}, & \omega(\theta)=1+\cos \theta, \\
\alpha=\frac{1}{2}, & \beta=-\frac{1}{2}, & \omega(\theta)=1-\cos \theta .
\end{array}
$$

These are three of the so-called Chebyshev weight functions, i.e., $\alpha, \beta \in\left\{ \pm \frac{1}{2}\right\}$. The remaining case $\alpha=\beta=-\frac{1}{2}$ gives rise to $\omega(\theta)=1$ (Lebesgue measure) where Szegö quadrature formulas are well known (see e.g., [5]).

On the other hand, taking advantage of the connection between orthogonal polynomials on the unit circle and the interval $[-1,1]$, in $[8$ it is proved that for the weight functions (3.1), the sequence $\rho_{n}(z)$ of monic polynomials satisfies

$$
\rho_{n}(0)=\frac{\alpha+\frac{1}{2}+(-1)^{n}\left(\beta+\frac{1}{2}\right)}{n+\alpha+\beta+1} .
$$


We will consider first the weight function: $\omega(\theta)=\frac{\sin ^{2}(\theta)}{2 \pi}$. Its moment sequence is given by $\mu_{0}=1 / 2, \mu_{1}=0, \mu_{2}=-1 / 4$ and $\mu_{k}=0 \forall k \geq 3$. From (3.3) the reflection coefficients are $\delta_{n}:=\rho_{n}(0)=\frac{1+(-1)^{n}}{n+2}$. The Szegö polynomials for some values of $n$ in this case are

$$
\begin{array}{ll}
\rho_{2}(z)=\frac{1}{2}+z^{2}, & \rho_{3}(z)=z\left(\frac{1}{2}+z^{2}\right), \\
\rho_{4}(z)=\frac{1}{3}+\frac{2}{3} z^{2}+z^{4}, & \rho_{5}(z)=z\left(\frac{1}{3}+\frac{2}{3} z^{2}+z^{4}\right) .
\end{array}
$$

As a general rule, we have deduced the following

Proposition 3.1. The sequence $\left\{\rho_{n}\right\}$, where

$$
\rho_{n}(z)= \begin{cases}\frac{2}{n+2} \sum_{i=0}^{n / 2}(i+1) z^{2 i} & \text { if } n \text { is even, } \\ z \frac{2}{n+1} \sum_{i=0}^{(n-1) / 2}(i+1) z^{2 i} & \text { if } n \text { is odd },\end{cases}
$$

is the sequence of monic Szegö polynomials with respect to the weight function $\omega(\theta)=\frac{\sin ^{2}(\theta)}{2 \pi}$, for all $n$.

Proof. Suppose that $n$ is even, then

$$
\begin{aligned}
\left\langle\rho_{n}(z), z^{k}\right\rangle_{\omega} & =\int_{0}^{2 \pi} \rho_{n}\left(e^{i \theta}\right) e^{-i k \theta} \frac{\sin ^{2}(\theta)}{2 \pi} d \theta \\
& =\frac{1}{2 \pi i} \int_{\mathbb{T}} \frac{\left(\frac{-1}{2 n+4} \sum_{i=0}^{n / 2}(i+1) z^{2 i}\right)\left(z^{2}-1\right)^{2}}{z^{k+3}} d z \\
& =\operatorname{Res}(h, 0),
\end{aligned}
$$

where $h(z)=\frac{\left(\frac{-1}{2 n+4} \sum_{i=0}^{n / 2}(i+1) z^{2 i}\right)\left(z^{2}-1\right)^{2}}{z^{k+3}}$. Therefore,

$$
h(z)=\frac{-1}{2 n+4}\left(\sum_{i=0}^{n / 2} \frac{i+1}{z^{k-2 i-1}}+\sum_{i=0}^{n / 2} \frac{-2(i+1)}{z^{k-2 i+1}}+\sum_{i=0}^{n / 2} \frac{i+1}{z^{k-2 i+3}}\right) .
$$

Note that if $k$ is odd, then $\operatorname{Res}(h, 0)=0$. So, if $k=0$, then

$$
\operatorname{Res}(h, 0)=\frac{-1}{2 n+4}(-2+2)=0 .
$$

If $2 \leq k \leq n-2$, then

$$
\operatorname{Res}(h, 0)=\frac{-1}{2 n+4}\left(\frac{k-2}{2}+1-2\left(\frac{k}{2}+1\right)+\frac{k+2}{2}+1\right)=0 .
$$

If $k=n$, then

$$
\operatorname{Res}(h, 0)=\frac{-1}{2 n+4}\left(\frac{n}{2}-n-2\right)=\frac{n+4}{4(n+2)} \neq 0 .
$$

If $n$ is odd, we have

$$
\begin{aligned}
\left\langle\rho_{n}(z), z^{k}\right\rangle_{\omega} & =\left\langle z \rho_{n-1}(z), z^{k}\right\rangle_{\omega} \\
& =\left\langle\rho_{n-1}(z), z^{k-1}\right\rangle_{\omega} \\
& =\left\{\begin{array}{lll}
0 & \text { if } \quad 1 \leq k \leq n-1 \\
\frac{n+4}{4(n+2)} & \text { if } \quad k=n .
\end{array}\right.
\end{aligned}
$$

If $k=0$,

$$
\begin{aligned}
\left\langle\rho_{n}(z), 1\right\rangle_{\omega} & =\frac{1}{2 \pi i} \int_{\mathbb{T}} \frac{z \rho_{n-1}(z)\left(z^{2}-1\right)^{2}}{-4 z^{3}} d z \\
& =\frac{1}{2 \pi i} \int_{\mathbb{T}} \frac{\rho_{n-1}(z)\left(z^{2}-1\right)^{2}}{-4 z^{2}} d z \\
& =\operatorname{Res}(h, 0),
\end{aligned}
$$


where

$$
\begin{aligned}
h(z)=\frac{-1}{2(n+1)}\left(\sum_{i=0}^{(n-1) / 2}(i+1)\left(z^{2 i+2}\right)+\right. & \sum_{i=0}^{(n-1) / 2}(-2(i+1))\left(z^{2 i}\right) \\
& \left.+\sum_{i=0}^{(n-1) / 2}(i+1)\left(z^{2 i-2}\right)\right) .
\end{aligned}
$$

Thus, $\operatorname{Res}(h, 0)=0$. Therefore, for all $n$, we have that

$$
\left\langle\rho_{n}(z), z^{k}\right\rangle_{\omega}=\left\{\begin{array}{lll}
0 & \text { if } \quad 0 \leq k \leq n-1, \\
\frac{n+4}{4(n+2)} & \text { if } \quad k=n
\end{array}\right.
$$

The associated polynomials for some values of $n$ are

$$
\begin{array}{rlrl}
\pi_{0}(z) & =-\frac{1}{2}, & \pi_{1}(z)=-\frac{1}{2} z \\
\pi_{2}(z)=\frac{1}{4}-\frac{1}{2} z^{2}, & \pi_{3}(z)=z\left(\frac{1}{4}-\frac{1}{2} z^{2}\right), \\
\pi_{4}(z)=\frac{1}{6}+\frac{1}{6} z^{2}-\frac{1}{2} z^{4}, & \pi_{5}(z)=z\left(\frac{1}{6}+\frac{1}{6} z^{2}-\frac{1}{2} z^{4}\right) .
\end{array}
$$

Proposition 3.2. The sequence $\left\{\pi_{n}\right\}$, where

$$
\begin{array}{ll}
\pi_{n}(z)=\frac{1}{n+2} \frac{1-z^{n}}{1-z^{2}}-\frac{1}{2} z^{n} & \text { for } n \text { even } \\
\pi_{n}(z)=z\left(\frac{1}{n+1} \frac{1-z^{n-1}}{1-z^{2}}-\frac{1}{2} z^{n-1}\right)=z \pi_{n-1}(z) & \text { for } n \text { odd }
\end{array}
$$

is the sequence of associated polynomials with respect to the weight function $\omega(\theta)=$ $\frac{\sin ^{2}(\theta)}{2 \pi}$, for all $n$.

Proof. For $n$ even,

$$
\begin{aligned}
z \pi_{n-1} & (z)-\delta_{n} \pi_{n-1}^{*}(z)=z^{2} \pi_{n-2}(z)-\delta_{n} \pi_{n-2}^{*}(z) \\
& =z^{2}\left(\frac{1}{n} \frac{1-z^{n-2}}{1-z^{2}}-\frac{1}{2} z^{n-2}\right)-\frac{2}{n+2}\left(\frac{1}{n} \frac{z^{2}\left(1-z^{n-2}\right)}{1-z^{2}}-\frac{1}{2}\right) \\
& =\frac{1}{n} \frac{z^{2}\left(1-z^{n-2}\right)}{1-z^{2}}-\frac{1}{n(n+2)} \frac{z^{2}\left(1-z^{n-2}\right)}{1-z^{2}}+\frac{1}{n+2}-\frac{1}{2} z^{n-2} \\
& =\frac{1}{n(n+2)\left(1-z^{2}\right)}\left((n+2) z^{2}\left(1-z^{n-2}\right)-2 z^{2}\left(1-z^{n-2}\right)+\left(1-z^{2}\right) n\right)-\frac{1}{2} z^{n} \\
& =\frac{1}{n(n+2)\left(1-z^{2}\right)}\left((n+2) z^{2}-(n+2) z^{n}-2 z^{2}+2 z^{n}+n-n z^{2}\right)-\frac{1}{2} z^{n} \\
& =\frac{1}{n(n+2)\left(1-z^{2}\right)}\left(-n z^{n}+n\right)-\frac{1}{2} z^{n} \\
& =\frac{1}{n+2} \frac{1-z^{n}}{1-z^{2}}-\frac{1}{2} z^{n} \\
& =\pi_{n}(z) .
\end{aligned}
$$


On the other hand,

$$
\begin{aligned}
\delta_{n} z \pi_{n-1}(z)-\pi_{n-1}^{*}(z)=\delta_{n} z^{2} \pi_{n-2}(z)-\pi_{n-2}^{*}(z) \\
\quad=\frac{2}{n+2} z^{2}\left(\frac{1}{n} \frac{1-z^{n-2}}{1-z^{2}}-\frac{1}{2} z^{n-2}\right)-\left(\frac{1}{n} \frac{z^{2}\left(1-z^{n-2}\right)}{1-z^{2}}-\frac{1}{2}\right) \\
\quad=\frac{2}{n(n+2)} \frac{z^{2}\left(1-z^{n-2}\right)}{1-z^{2}}-\frac{1}{n} \frac{z^{2}\left(1-z^{n-2}\right)}{1-z^{2}}-\frac{1}{n+2} z^{n}+\frac{1}{2} \\
\quad=\frac{1}{n(n+2)\left(1-z^{2}\right)}\left(2 z^{2}\left(1-z^{n-2}\right)-(n+2) z^{2}\left(1-z^{n-2}\right)-\left(1-z^{2}\right) n z^{n}\right)+\frac{1}{2} \\
=\frac{1}{n(n+2)\left(1-z^{2}\right)}\left(-n z^{2}+n z^{n}-n z^{n}+n z^{n+2}\right)+\frac{1}{2} \\
=\frac{1}{n(n+2)\left(1-z^{2}\right)}\left(-n z^{2}\left(1-z^{n}\right)\right)+\frac{1}{2} \\
=-\frac{1}{n+2} \frac{z^{2}\left(1-z^{n}\right)}{1-z^{2}}+\frac{1}{2} \\
=-\pi_{n}^{*}(z) .
\end{aligned}
$$

For $n$ odd we have, since $\delta_{n}=0$,

$$
\begin{gathered}
z \pi_{n-1}(z)-\delta_{n} \pi_{n-1}^{*}(z)=z \pi_{n-1}(z)=\pi_{n}(z), \\
\delta_{n} z \pi_{n-1}(z)-\pi_{n-1}^{*}(z)=-\pi_{n-1}^{*}(z)=-\pi_{n}^{*}(z) .
\end{gathered}
$$

We have proved that the polynomial $\pi_{n}$, defined above, satisfies the recurrence relations

$$
\begin{gathered}
z \pi_{n-1}(z)-\delta_{n} \pi_{n-1}^{*}(z)=\pi_{n}^{*}(z), \\
\delta_{n} z \pi_{n-1}(z)-\pi_{n-1}^{*}(z)=-\pi_{n}^{*}(z) .
\end{gathered}
$$

Thus, since $\pi_{0}(z)=-\frac{1}{2}$, this proves the proposition.

If $\left\{\rho_{n}\right\}$ is the sequence of monic Szegö polynomials given in formula (3.4), we can construct the para-orthogonal polynomials $B_{n}\left(z, \tau_{n}\right)=\rho_{n}(z)+\tau_{n} \rho_{n}^{*}(z)$, where $\left|\tau_{n}\right|=1$. We have calculated them for $\tau_{n}=1$ and $\tau_{n}=-1, \forall n$ as given in the following

\section{Proposition 3.3.}

$$
B_{2 n}(z, 1)=\frac{(n+2)}{n+1} \frac{1-z^{2 n+2}}{1-z^{2}}, \quad B_{2 n}(z,-1)=\frac{1}{n+1} \sum_{i=0}^{n}(2 i-n) z^{2 i}
$$

and

$$
\begin{aligned}
& B_{2 n+1}(z, 1)=\frac{1}{n+1}\left(\sum_{i=0}^{n}(i+1) z^{2 i+1}+\sum_{i=0}^{n}(n-i+1) z^{2 i}\right), \\
& B_{2 n+1}(z,-1)=\frac{1}{n+1}\left(\sum_{i=0}^{n}(i+1) z^{2 i+1}-\sum_{i=0}^{n}(n-i+1) z^{2 i}\right) .
\end{aligned}
$$

Proof.

$$
\begin{aligned}
B_{2 n}(z, 1) & =\frac{1}{n+1} \sum_{i=0}^{n}(i+1) z^{2 i}+\frac{1}{n+1} \sum_{i=0}^{n}(n-i+1) z^{2 i} \\
& =\frac{1}{n+1} \sum_{i=0}^{n}((i+1)+(n-i+1)) z^{2 i} \\
& =\frac{n+2}{n+1} \sum_{i=0}^{n} z^{2 i} \\
& =\frac{(n+2)}{n+1} \frac{1-z^{2 n+2}}{1-z^{2}}
\end{aligned}
$$


Similarly,

$$
\begin{aligned}
B_{2 n}(z,-1) & =\frac{1}{n+1} \sum_{i=0}^{n}(i+1) z^{2 i}-\frac{1}{n+1} \sum_{i=0}^{n}(n-i+1) z^{2 i} \\
& =\frac{1}{n+1} \sum_{i=0}^{n}((i+1)-(n-i+1)) z^{2 i} \\
& =\frac{1}{n+1} \sum_{i=0}^{n}(2 i-n) z^{2 i}
\end{aligned}
$$

and

$$
\begin{aligned}
B_{2 n+1}(z, 1) & =\rho_{2 n+1}(z)+\rho_{2 n+1}^{*}(z)=z \rho_{2 n}(z)+\rho_{2 n}^{*}(z) \\
& =\frac{1}{n+1} \sum_{i=0}^{n}(i+1) z^{2 i+1}+\frac{1}{n+1} \sum_{i=0}^{n}(n-i+1) z^{2 i} \\
& =\frac{1}{n+1}\left(\sum_{i=0}^{n}(i+1) z^{2 i+1}+\sum_{i=0}^{n}(n-i+1) z^{2 i}\right), \\
B_{2 n+1}(z,-1) & =\rho_{2 n+1}(z)-\rho_{2 n+1}^{*}(z)=z \rho_{2 n}(z)-\rho_{2 n}^{*}(z) \\
& =\frac{1}{n+1} \sum_{i=0}^{n}(i+1) z^{2 i+1}-\frac{1}{n+1} \sum_{i=0}^{n}(n-i+1) z^{2 i} \\
& =\frac{1}{n+1}\left(\sum_{i=0}^{n}(i+1) z^{2 i+1}-\sum_{i=0}^{n}(n-i+1) z^{2 i}\right) .
\end{aligned}
$$

Similarly, we can construct the polynomial $A_{n}\left(z, \tau_{n}\right)=\pi_{n}(z)-\tau_{n} \pi_{n}^{*}(z)$, where again, $\left|\tau_{n}\right|=1, \forall n$. If the sequence $\left\{\pi_{n}\right\}$ is given by formula (3.5), then we have

\section{Proposition 3.4.}

$$
A_{2 n}(z, 1)=\frac{n+2}{2(n+1)}\left(1-z^{2 n}\right), \quad A_{2 n}(z,-1)=\frac{n\left(z^{2 n+2}-1\right)}{2(n+1)\left(1-z^{2}\right)}+\frac{(n+2) z^{2}\left(1-z^{2 n-2}\right)}{2(n+1)\left(1-z^{2}\right)}
$$

and

$$
\begin{aligned}
& A_{2 n+1}(z, 1)=\frac{z\left(1-z^{2 n}\right)}{2(n+1)(1+z)}+\frac{1}{2}\left(1-z^{2 n+1}\right), \\
& A_{2 n+1}(z,-1)=\frac{z\left(1-z^{2 n}\right)}{2(n+1)(1-z)}-\frac{1}{2}\left(1+z^{2 n+1}\right) .
\end{aligned}
$$

Proof.

$$
\begin{aligned}
A_{2 n}( & z, 1)=\left(\frac{1}{2 n+2} \frac{1-z^{2 n}}{1-z^{2}}-\frac{1}{2} z^{2 n}\right)-\left(\frac{1}{2 n+2} \frac{z^{2}\left(1-z^{2 n}\right)}{1-z^{2}}-\frac{1}{2}\right) \\
& =\frac{1}{2(2 n+2)\left(1-z^{2}\right)}\left(2\left(1-z^{2 n}\right)-2 z^{2}\left(1-z^{2 n}\right)+\left(1-z^{2 n}\right)(2 n+2)\left(1-z^{2}\right)\right) \\
& =\frac{1}{2(2 n+2)\left(1-z^{2}\right)}\left((2 n+4)-(2 n+4) z^{2}-(2 n+4) z^{2 n}+(2 n+4) z^{2 n+2}\right) \\
& =\frac{n+4}{2(2 n+2)\left(1-z^{2}\right)}\left(1-z^{2}-z^{2 n}+z^{2 n+2}\right) \\
& =\frac{2 n+4}{2(2 n+2)\left(1-z^{2}\right)}\left(\left(1-z^{2}\right)\left(1-z^{2 n}\right)\right) \\
& =\frac{n+2}{2(n+1)}\left(1-z^{2 n}\right), \\
A_{2 n}( & (-1)=\left(\frac{1}{2 n+2} \frac{1-z^{2 n}}{1-z^{2}}-\frac{1}{2} z^{2 n}\right)+\left(\frac{1}{2 n+2} \frac{z^{2}\left(1-z^{2 n}\right)}{1-z^{2}}-\frac{1}{2}\right) \\
& =\frac{1}{2(2 n+2)\left(1-z^{2}\right)}\left(2\left(1-z^{2 n}\right)+2 z^{2}\left(1-z^{2 n}\right)-\left(1+z^{2 n}\right)(2 n+2)\left(1-z^{2}\right)\right) \\
& =\frac{1}{2(2 n+2)\left(1-z^{2}\right)}\left(-2 n-(2 n+4) z^{2}-(2 n+4) z^{2 n}+2 n z^{2 n+2}\right) \\
& =\frac{n\left(z^{2 n+2}-1\right)}{2(n+1)\left(1-z^{2}\right)}+\frac{(n+2) z^{2}\left(1-z^{2 n-2}\right)}{2(n+1)\left(1-z^{2}\right)} .
\end{aligned}
$$


Similarly,

$$
\begin{aligned}
& A_{2 n+1}(z, 1)=\pi_{2 n+1}(z)-\pi_{2 n+1}^{*}(z)=z \pi_{2 n}(z)-\pi_{2 n}^{*}(z) \\
& \quad=z\left(\frac{1}{2(n+1)} \frac{1-z^{2 n}}{1-z^{2}}-\frac{1}{2} z^{2 n}\right)-\left(\frac{1}{2(n+1)} \frac{z^{2}\left(1-z^{2 n}\right)}{1-z^{2}}-\frac{1}{2}\right) \\
& \quad=\frac{1}{2(n+1)\left(1-z^{2}\right)}\left(z\left(1-z^{2 n}\right)-z^{2}\left(1-z^{2 n}\right)+\left(1-z^{2 n+1}\right)(n+1)\left(1-z^{2}\right)\right) \\
& \quad=\frac{1}{2(n+1)\left(1-z^{2}\right)}\left(z\left(1-z^{2 n}\right)(1-z)+\left(1-z^{2 n+1}\right)(n+1)\left(1-z^{2}\right)\right) \\
& \quad=\frac{z\left(1-z^{2 n}\right)}{2(n+1)(1+z)}+\frac{1}{2}\left(1-z^{2 n+1}\right), \\
& A_{2 n+1}(z,-1)=\pi_{2 n+1}(z)+\pi_{2 n+1}^{*}(z)=z \pi_{2 n}(z)+\pi_{2 n}^{*}(z) \\
& \quad=z\left(\frac{1}{2(n+1)} \frac{1-z^{2 n}}{1-z^{2}}-\frac{1}{2} z^{2 n}\right)+\left(\frac{1}{2(n+1)} \frac{z^{2}\left(1-z^{2 n}\right)}{1-z^{2}}-\frac{1}{2}\right) \\
& \quad=\frac{1}{2(n+1)\left(1-z^{2}\right)}\left(z\left(1-z^{2 n}\right)+z^{2}\left(1-z^{2 n}\right)-\left(1+z^{2 n+1}\right)(n+1)\left(1-z^{2}\right)\right) \\
& \quad=\frac{1}{2(n+1)\left(1-z^{2}\right)}\left(z\left(1-z^{2 n}\right)(1+z)-\left(1+z^{2 n+1}\right)(n+1)\left(1-z^{2}\right)\right) \\
& \quad=\frac{z\left(1-z^{2 n}\right)}{2(n+1)(1-z)}-\frac{1}{2}\left(1+z^{2 n+1}\right) .
\end{aligned}
$$

We know that the coefficients of the Szegö quadrature formula can be written as in (2.7). If $A_{n}$ and $B_{n}$ are as in formulas (3.8) and (3.6), respectively, for $n$ even, we have

$$
\begin{aligned}
B_{n}^{\prime}(z, 1) & =\frac{n+4}{n+2} \frac{-(n+2) z^{n+1}\left(1-z^{2}\right)-\left(1-z^{n+2}\right)(-2 z)}{\left(1-z^{2}\right)^{2}} \\
& =\frac{n+4}{n+2} \frac{-(n+2) z^{n+1}+(n+2) z^{n+3}+2 z-2 z^{n+3}}{(1+z)^{2}} \\
& =\frac{n+4}{n+2} z\left(\frac{2-(n+2) z^{n}+n z^{n+2}}{\left(1-z^{2}\right)^{2}}\right) .
\end{aligned}
$$

In this case, the nodes are $x_{k}=e^{\frac{2 k \pi i}{n+2}}, k=1, \ldots, n+1, k \neq \frac{n}{2}+1$. Thus, for all $k=1, \ldots, n+1, k \neq \frac{n}{2}+1$ and by formula (2.7),

$$
\begin{aligned}
\lambda_{k} & =\frac{-1}{2 e^{\frac{2 k \pi i}{n+2}}} \frac{1-\left(-e^{\frac{2 k \pi i}{n+2}}\right)^{n}}{\frac{2 e^{\frac{2 k \pi i}{n+2}}\left(-(n+2)\left(e^{\frac{2 k \pi i}{n+2}}\right)^{n}+n+2\right)}{\left(1-e^{\frac{4 k \pi i}{n+2}}\right)^{2}}} \\
& =-\frac{1}{4 e^{\frac{2 k \pi i}{n+2}}} \frac{1-e^{\frac{2 k n \pi i}{n+2}}}{(n+2)\left(1-e^{\frac{2 k n \pi i}{n+2}}\right)}\left(1-e^{\frac{4 k n \pi i}{n+2}}\right)^{2} \\
& =-\frac{1}{4(n+2)} \frac{1-2 e^{\frac{4 k \pi i}{n+2}}+e^{\frac{8 k \pi i}{n+2}}}{e^{\frac{4 k \pi i}{n+2}}} \\
& =-\frac{1}{4(n+2)}\left(-2+2 \Re\left(e^{\frac{4 k \pi i}{n+2}}\right)\right) \\
& =\frac{1-\cos \left(\frac{4 k \pi}{n+2}\right)}{2(n+2)} .
\end{aligned}
$$

In short, the following theorem has been shown

Theorem 3.5. The coefficients of the n-point Szegö quadrature formula for $n$ even corresponding to $\tau_{n}=1, n=1,2, \ldots$ for the weight function $\omega(\theta)=\frac{\sin ^{2} \theta}{2 \pi}$ are

$$
\lambda_{k}=\frac{1-\cos \left(\frac{4 k \pi}{n+2}\right)}{2(n+2)}, k=1, \ldots, n+1, k \neq \frac{n}{2}+1,
$$

and the nodes are given by $x_{k}=e^{\frac{2 k \pi i}{n+2}}, k=1, \ldots, n+1, k \neq \frac{n}{2}+1$. 
When $\tau_{n} \neq 1$ explicit formulas for the nodes and the weights are not available. For the purpose of illustration we have calculated the weights and nodes for the $n$-point Szegö quadrature formula for some values of even $n$ and $\tau_{n}=-1$. If we choose $n=4$, then

$$
B_{4}(z,-1)=\frac{2}{3}\left(z^{4}-1\right) \quad \text { and } \quad A_{4}(z,-1)=-\frac{1}{3}\left(z^{4}-z^{2}+1\right) .
$$

Thus, the nodes $\left\{x_{k}\right\}_{k=1}^{4}$ and the weights $\left\{\lambda_{k}\right\}_{k=1}^{4}$ will be given by

$$
\begin{aligned}
& x_{1}=1, \quad x_{2}=-1, \quad x_{3}=i, \quad x_{4}=-i, \\
& \lambda_{1}=\lambda_{2}=0.0625, \quad \lambda_{3}=\lambda_{4}=0.1875 .
\end{aligned}
$$

If we take $n=6$, then in this case we have

$$
B_{6}(z,-1)=\frac{1}{4}\left(3 z^{6}+z^{4}-z^{2}-3\right) \quad \text { and } \quad A_{6}(z,-1)=-\frac{1}{8}\left(3 z^{6}-2 z^{4}-2 z^{2}+3\right),
$$

and the nodes $\left\{x_{k}\right\}_{k=1}^{6}$ and the weights $\left\{\lambda_{k}\right\}_{k=1}^{6}$ will be given by

$$
\begin{array}{cl}
x_{1}=1, & x_{2}=-1, \\
x_{3}=0.408248+0.912871 i, & x_{4}=0.408248-0.912871 i, \\
x_{5}=-0.408248-0.912871 i, & x_{6}=-0.408248+0.912871 i, \\
& \text { if } \quad k=1,2, \\
\lambda_{k}=0.025 & \text { if } \quad 3 \leq k \leq 6 .
\end{array}
$$

In order to obtain the upper bound given in formula (2.10) and the second kind measure associated with the weight function $\omega(\theta)=\frac{\sin ^{2} \theta}{2 \pi}$, we have calculated the Herglotz-Riesz transform

$$
\begin{aligned}
F_{\omega}(z) & =\int_{0}^{2 \pi} \frac{e^{i \theta}+z}{e^{i \theta}-z} \frac{\sin ^{2}(\theta)}{2 \pi} d \theta \\
& =\frac{1}{2 \pi i} \int_{\mathbb{T}} \frac{-(w+z)\left(w^{2}-1\right)^{2}}{4 w^{3}(w-z)} d w \\
& =\left\{\begin{array}{lll}
\operatorname{Res}(h, 0)+\operatorname{Res}(h, z) & \text { if } & |z|<1, \\
\operatorname{Res}(h, 0) & \text { if } & |z|>1,
\end{array}\right.
\end{aligned}
$$

where $h(w)=\frac{-(w+z)\left(w^{2}-1\right)^{2}}{4 w^{3}(w-z)}$. Since

$$
\frac{1}{w-z}=\sum_{i=0}^{\infty}\left(\frac{-1}{z^{i+1}}\right) w^{i}
$$

and

$$
\frac{-(w+z)\left(w^{2}-1\right)^{2}}{4 w^{3}(w-z)}=\frac{-w^{2}}{4}+\frac{1}{2}+\frac{-1}{4 w^{2}}+\frac{-z w}{4}+\frac{z}{2 w}+\frac{-z}{4 w^{3}},
$$

then

$$
\operatorname{Res}(h, 0)=\frac{1}{4 z^{2}}+\frac{-z}{2 z}+\frac{z}{4 z^{3}}=\frac{1-z^{2}}{2 z^{2}} \quad \text { and } \quad \operatorname{Res}(h, z)=\frac{-2 z\left(z^{2}-1\right)^{2}}{4 z^{3}}=\frac{-\left(z^{2}-1\right)^{2}}{2 z^{2}} .
$$

Therefore

$$
F_{\omega}(z)=\left\{\begin{array}{lll}
\frac{1-z^{2}}{2} & \text { if } & |z|<1, \\
\frac{1-z^{2}}{2 z^{2}} & \text { if } & |z|>1 .
\end{array}\right.
$$

In short, we have the following 
Proposition 3.6. The Herglotz-Riesz transform associated with the weight function $\omega(\theta)=\frac{\sin ^{2} \theta}{2 \pi}$ is given by

$$
F_{\omega}(z)=\left\{\begin{array}{lll}
\frac{1-z^{2}}{2} & \text { if } & |z|<1 \\
\frac{1-z^{2}}{2 z^{2}} & \text { if } & |z|>1
\end{array}\right.
$$

Then we have

Corollary 3.7. $d \widetilde{\psi}=d \theta+\pi\left(d \delta\left(e^{i \theta}-z_{1}\right)+d \delta\left(e^{i \theta}-z_{2}\right)\right)$ is the second kind measure associated with $d \psi(\theta)=\frac{\sin ^{2} \theta}{2 \pi} d \theta$, where $z_{1}=1$ and $z_{2}=-1$.

Proof. By (3.10), if $|z|<1$, then $F(z)=\frac{1-z^{2}}{2}$ is the Carathéodory function corresponding to the measure $d \psi(\theta)=\frac{\sin ^{2} \theta}{2 \pi} d \theta$ and then, by Theorem 1.3, $G(z)=\frac{2}{1-z^{2}}$ is the Carathéodory function corresponding to the measure $\widetilde{\psi}$ given in Theorem 1.2. By formula (2.11),

$$
\begin{aligned}
& z_{1}=1, \gamma_{1}=-1, \\
& z_{2}=-1, \gamma_{2}=1
\end{aligned}
$$

and

$$
\begin{aligned}
G\left(e^{i \theta}\right) & =2 \frac{1}{1-\cos 2 \theta-i \sin 2 \theta}=2 \frac{1-\cos 2 \theta+i \sin 2 \theta}{2-2 \cos 2 \theta} \\
& =1+\frac{\sin 2 \theta}{1-\cos 2 \theta} i .
\end{aligned}
$$

Thus, $\Re\left(G\left(e^{i \theta}\right)\right)=1$ and, by formula (2.12),

$$
d \widetilde{\psi}=d \theta+\pi\left(d \delta\left(e^{i \theta}-z_{1}\right)+d \delta\left(e^{i \theta}-z_{2}\right)\right)
$$

and the sequence $\left\{\pi_{n}\right\}$, given as in formula (3.5), is orthogonal with respect to $d \widetilde{\psi}$.

If $A_{n}$ and $B_{n}$ are as in formulas (3.8) and (3.6), respectively, for $n$ even, we can compute the modified approximants: $R_{n}(z, 1)=\frac{A_{n}(z, 1)}{B_{n}(z, 1)}$ and we obtain

$$
R_{n}(z, 1)=\frac{\left(1-z^{n}\right)\left(1-z^{2}\right)}{2\left(1-z^{n+2}\right)}
$$

and the error for the Herglotz-Riesz transform is given by

$$
F_{\omega}(z)-R_{n}(z, 1)=\left\{\begin{array}{lll}
\frac{z^{n}\left(1-z^{2}\right)^{2}}{2\left(1-z^{n+2}\right)} & \text { if } & |z|<1, \\
\frac{\left(1-z^{2}\right)^{2}}{2 z^{2}\left(1-z^{n+2}\right)} & \text { if } & |z|>1 .
\end{array}\right.
$$

According to Lemma 2.1, if $G=\{z: r<|z|<R, r<1, R>1\}$, then $\Gamma=$ $\Gamma_{1} \cup \Gamma_{2}$, where

$$
\Gamma_{1}=\{z:|z|=r, 0<r<1\} \quad \text { and } \quad \Gamma_{2}=\{z:|z|=R, R>1\} .
$$

In this case, by formula (3.12) and (2.10)

$$
\begin{aligned}
\left|E_{n}(f)\right| & \leq \frac{1}{4 \pi} \max _{\xi \in \Gamma}\left\{\left|\frac{f(\xi)}{\xi}\right|\right\}\left(\int_{\Gamma_{1}} \frac{|z|^{n}\left|1-z^{2}\right|^{2}}{\left.2|1-| z\right|^{n+2}}+\int_{\Gamma_{2}} \frac{\left|1-z^{2}\right|^{2}}{2|z|^{2}\left(1-|z|^{n+2}\right)}\right) \\
= & \frac{1}{4 \pi} \max _{\xi \in \Gamma}\left\{\left|\frac{f(\xi)}{\xi}\right|\right\}\left(\int_{0}^{2 \pi} \frac{r^{n}\left(1-2 r^{2} \cos 2 \theta+r^{4}\right)}{2\left(1-r^{n+2}\right)} r d \theta+\int_{0}^{2 \pi} \frac{1-2 R^{2} \cos 2 \theta+R^{4}}{2 R^{2}\left(R^{n+2}-1\right)} R d \theta\right) \\
= & \frac{1}{4} \max _{\xi \in \Gamma}\left\{\left|\frac{f(\xi)}{\xi}\right|\right\}\left(\frac{r^{n+1}\left(1+r^{4}\right)}{1-r^{n+2}}+\frac{\left(1+R^{4}\right)}{R\left(R^{n+2}-1\right)}\right) .
\end{aligned}
$$

Thus, we have proved the following 
Theorem 3.8. Let $f$ be analytic in $G=\{z: r<|z|<R, r<1, R>1\}$. Then, for each $n$ even

$$
\left|E_{n}(f)\right| \leq \frac{1}{4} \max _{\xi \in \Gamma}\left\{\left|\frac{f(\xi)}{\xi}\right|\right\}\left(\frac{r^{n+1}\left(1+r^{4}\right)}{1-r^{n+2}}+\frac{\left(1+R^{4}\right)}{R\left(R^{n+2}-1\right)}\right),
$$

where $\Gamma=\Gamma_{1} \cup \Gamma_{2} . \Gamma_{1}$ and $\Gamma_{2}$ as in formula (3.13).

Remark 3.9. Note that if $f(z)$ is analytic in $\{z:|z|<R ; R>1\}$, then we can make $r \rightarrow 0$ and

$$
\left|E_{n}(f)\right| \leq \frac{1}{4} \max _{\xi \in \Gamma_{2}}\left\{\left|\frac{f(\xi)}{\xi}\right|\right\}\left(\frac{\left(1+R^{4}\right)}{R\left(R^{n+2}-1\right)}\right) .
$$

On the other hand, if $f(z)$ is analytic in $\{z: r<|z| \leq \infty ; 0<r<1\}$, now we can make $R \rightarrow \infty$ and one has

$$
\left|E_{n}(f)\right| \leq \frac{1}{4} \max _{\xi \in \Gamma_{1}}\left\{\left|\frac{f(\xi)}{\xi}\right|\right\}\left(\frac{r^{n+1}\left(1+r^{4}\right)}{1-r^{n+2}}\right) .
$$

Since for the remaining Chebyshev weight functions, i.e., $\omega(\theta)=\frac{1+\cos \theta}{2 \pi}$ and $\omega(\theta)=\frac{1-\cos \theta}{2 \pi}$, the calculations are quite similar to the previous case, we will omit them and give only the results.

First, we consider the Chebyshev weight function: $\omega(\theta)=\frac{1+\cos \theta}{2 \pi}$. In this case the moment sequence is given by $\mu_{0}=1, \mu_{1}=1 / 2$ and $\mu_{k}=0, \forall k \geq 2$. From (3.3), the reflection coefficients are $\delta_{n}:=\frac{(-1)^{n}}{n+1}, \forall n$ and we have the following expression for the Szegö polynomials

Proposition 3.10. The sequence $\left\{\rho_{n}\right\}$, given by

$$
\rho_{n}(z)=\frac{(-1)^{n}}{n+1} \sum_{i=0}^{n}(-1)^{i}(i+1) z^{i}
$$

is the sequence of monic Szegö polynomials with respect to the weight function $\omega(\theta)=\frac{1+\cos \theta}{2 \pi}$, for all $n$.

For the associated Szegö polynomials one has

Proposition 3.11. The sequence $\left\{\pi_{n}\right\}$, where

$$
\pi_{n}(z)=\frac{(-1)^{n}}{n+1} \frac{1+(-1)^{n-1} z^{n}}{1+z}-z^{n}
$$

is the sequence of associated polynomials with respect to the weight function $\omega(\theta)=$ $\frac{1+\cos \theta}{2 \pi}$, for all $n$.

Let $\left\{\rho_{n}\right\}$ be the sequence of monic Szegö polynomials given in formula (3.15). We have calculated the para-orthogonal polynomial $B_{n}\left(z, \tau_{n}\right)$ for $\tau_{n}=(-1)^{n}, \forall n$ and $\tau_{n}=(-1)^{n+1}, \forall n$ :

\section{Proposition 3.12.}

$$
B_{n}\left(z,(-1)^{n}\right)=\frac{(-1)^{n}(n+2)}{n+1} \frac{1+(-1)^{n} z^{n+1}}{1+z}
$$

and

$$
B_{n}\left(z,(-1)^{n+1}\right)=\frac{(-1)^{n}}{n+1} \sum_{i=0}^{n}(-1)^{i}(2 i-n) z^{i}
$$


Similarly, if the sequence $\left\{\pi_{n}\right\}$ is given by formula (3.16) , for $A_{n}\left(z,(-1)^{n}\right)$ and $A_{n}\left(z,(-1)^{n+1}\right)$ we have the following

Proposition 3.13.

$$
A_{n}\left(z,(-1)^{n}\right)=\frac{(-1)^{n}(n+2)}{n+1}\left(1-(-1)^{n} z^{n}\right)
$$

and

$$
A_{n}\left(z,(-1)^{n+1}\right)=\frac{(-1)^{n} n\left(1-(-1)^{n+1} z^{n+1}\right)}{(n+1)(1+z)}+\frac{(-1)^{n}(n+2) z\left(1+(-1)^{n} z^{n-1}\right)}{(n+1)(1+z)} .
$$

If we choose $B_{n}$ as in formula (3.17) and $A_{n}$ as in formula (3.19), we have,

Theorem 3.14. The coefficients of the n-point Szegö quadrature formula corresponding to $\tau_{n}=(-1)^{n}$ for the weight function $\omega(\theta)=\frac{1+\cos \theta}{2 \pi}$ are

$$
\lambda_{k}= \begin{cases}\frac{1+\cos \left(\frac{2 k \pi}{n+1}\right)}{n+1} & \text { if } n \text { is odd, } 0 \leq k \leq n, k \neq \frac{n+1}{2}, \\ \frac{1+\cos \left(\frac{\pi+2 k \pi}{n+1}\right)}{n+1} & \text { if } n \text { is even, } 0 \leq k \leq n, k \neq \frac{n}{2},\end{cases}
$$

and the nodes are given by

$$
x_{k}= \begin{cases}e^{\frac{2 k \pi i}{n+1}} & \text { if } n \text { is odd, } 0 \leq k \leq n, k \neq \frac{n+1}{2}, \\ e^{\frac{(\pi+2 k \pi) i}{n+1}} & \text { if } n \text { is even, } 0 \leq k \leq n, k \neq \frac{n}{2} .\end{cases}
$$

In order to obtain the upper bound given in formula (2.10) and the second kind measure associated with the weight function $\omega(\theta)=\frac{1+\cos \theta}{2 \pi}$, we have calculated the Herglotz-Riesz transform:

Proposition 3.15. The Herglotz-Riesz transform associated with the weight function $\omega(\theta)=\frac{1+\cos \theta}{2 \pi}$ is given by

$$
F_{\omega}(z)=\left\{\begin{array}{cll}
1+z & \text { if } & |z|<1 \\
-\frac{1+z}{z} & \text { if } & |z|>1
\end{array}\right.
$$

As a corollary of this proposition we have

Corollary 3.16. $d \widetilde{\psi}=\frac{1}{2} d \theta+\pi d \delta\left(e^{i \theta}-z_{1}\right)$ is the second kind measure associated with $d \psi(\theta)=\frac{1+\cos \theta}{2 \pi} d \theta$, where $z_{1}=-1$.

If $A_{n}$ and $B_{n}$ are as in formulas (3.19) and (3.17), respectively, we can compute the modified approximants: $R_{n}\left(z,(-1)^{n}\right)=\frac{A_{n}\left(z,(-1)^{n}\right)}{B_{n}\left(z,(-1)^{n}\right)}$ and we obtain

$$
R_{n}\left(z,(-1)^{n}\right)=\frac{\left(1-(-1)^{n} z^{n}\right)(1+z)}{1+(-1)^{n} z^{n+1}}
$$

and the error for the Herglotz-Riesz transform is given by

$$
F_{\omega}(z)-R_{n}\left(z,(-1)^{n}\right)=\left\{\begin{array}{lll}
\frac{(-1)^{n} z^{n}(1+z)^{2}}{1+(-1)^{n} z^{n+1}} & \text { if } & |z|<1 \\
\frac{-(1+z)^{2}}{z\left(1+(-1)^{n} z^{n+1}\right)} & \text { if } & |z|>1
\end{array}\right.
$$

In this case, by formula $(\underline{3.23})$ and (2.10) 
Theorem 3.17. Let $f$ be analytic in $G=\{z: r<|z|<R, r<1, R>1\}$. Then, for each $n$

$$
\left|E_{n}(f)\right| \leq \frac{1}{2} \max _{\xi \in \Gamma}\left\{\left|\frac{f(\xi)}{\xi}\right|\right\}\left(\frac{r^{n+1}\left(1+r^{2}\right)}{1-r^{n+1}}+\frac{\left(1+R^{2}\right)}{R^{n+1}-1}\right),
$$

where $\Gamma=\Gamma_{1} \cup \Gamma_{2} . \Gamma_{1}$ and $\Gamma_{2}$ as in formula 3.13).

For this weight function a result similar to that given in Remark 3.9 also holds.

Finally, for the Chebyshev weight function $\omega(\theta)=\frac{1-\cos \theta}{2 \pi}$, we have the following: its moment sequence is given by $\mu_{0}=1, \mu_{1}=-1 / 2$ and $\mu_{k}=0 \forall k \geq 2$. Note that in this case, from (3.3), the reflection coefficients are $\delta_{n}:=\rho_{n}(0)=\frac{1}{n+1}, \forall n$.

Proposition 3.18. The sequence $\left\{\rho_{n}\right\}$, where

$$
\rho_{n}(z)=\frac{1}{n+1} \sum_{i=0}^{n}(i+1) z^{i}
$$

is the sequence of monic Szegö polynomials with respect to the weight function $\omega(\theta)=\frac{1-\cos \theta}{2 \pi}$, for all $n$.

Remark 3.19. Proceeding as in proof of Proposition 3.1, it can be checked that

$$
\left\langle\rho_{n}(z), z^{k}\right\rangle_{\omega}=0, \quad 0 \leq k \leq n-1 .
$$

However, the weight function under consideration is a particular case of $\left|\sin \frac{\theta}{2}\right|^{2 \alpha}$, $\alpha>-\frac{1}{2}$ where the sequence $\left\{\rho_{n}(z)\right\}$ of monic orthogonal polynomials is explicitly known. Indeed, it holds 8 that

$$
\rho_{n}(z)=\sum_{k=0}^{n}\left(\begin{array}{c}
n \\
k
\end{array}\right) \frac{\Gamma(\alpha+n-k) \Gamma(\alpha+k+1)}{\Gamma(\alpha+n+1) \Gamma(\alpha)} z^{k} .
$$

Thus, when taking $\alpha=1$ in (3.26), formula (3.25) follows.

The associated polynomials are now given in the following

Proposition 3.20. The sequence $\left\{\pi_{n}\right\}$, where

$$
\pi_{n}(z)=\frac{1}{n+1} \frac{1-z^{n}}{1-z}-z^{n}
$$

is the sequence of associated polynomials with respect to the weight function $\omega(\theta)=$ $\frac{1-\cos \theta}{2 \pi}$, for all $n$.

If $\left\{\rho_{n}\right\}$ is the sequence of monic Szegö polynomials given in formula (3.25), the para-orthogonal polynomials $B_{n}\left(z, \tau_{n}\right)$ for $\tau_{n}=1, \forall n$ and $\tau_{n}=-1, \forall n$, are given by

\section{Proposition 3.21.}

$$
B_{n}(z, 1)=\frac{(n+2)}{n+1} \frac{1-z^{n+1}}{1-z}
$$

and

$$
B_{n}(z,-1)=\frac{1}{n+1} \sum_{i=0}^{n}(2 i-n) z^{i}
$$

If the sequence $\left\{\pi_{n}\right\}$ is given by formula (3.27), then, for $A_{n}(z, 1)$ and $A_{n}(z,-1)$ one can write 
Proposition 3.22.

$$
A_{n}(z, 1)=\frac{n+2}{n+1}\left(1-z^{n}\right)
$$

and

$$
A_{n}(z,-1)=\frac{n\left(z^{n+1}-1\right)}{(n+1)(1-z)}+\frac{(n+2) z\left(1-z^{n-1}\right)}{(n+1)(1-z)} .
$$

If we choose $B_{n}$ as in formula (3.28) and $A_{n}$ as in formula (3.30), we have,

Theorem 3.23. The coefficients of the n-point Szegö quadrature formula corresponding to $\tau_{n}=1$ for the weight function $\omega(\theta)=\frac{1-\cos \theta}{2 \pi}$ are

$$
\lambda_{k}=\frac{1-\cos \left(\frac{2 k \pi}{n+1}\right)}{n+1}
$$

and the nodes are $x_{k}=e^{\frac{2 k \pi i}{n+1}}, k=1, \ldots, n$.

For the weight function $\omega(\theta)=\frac{1-\cos \theta}{2 \pi}$, the Herglotz-Riesz transform is given by

Proposition 3.24. The Herglotz-Riesz transform associated with the weight function $\omega(\theta)=\frac{1-\cos \theta}{2 \pi}$ is given by

$$
F_{\omega}(z)=\left\{\begin{array}{lll}
1-z & \text { if } & |z|<1 \\
\frac{1-z}{z} & \text { if } & |z|>1
\end{array}\right.
$$

Again we have

Corollary 3.25. $d \widetilde{\psi}=\frac{1}{2} d \theta+\pi d \delta\left(e^{i \theta}-z_{1}\right)$ is the second kind weight function associated with $d \psi(\theta)=\frac{1-\cos \theta}{2 \pi} d \theta$, where $z_{1}=1$.

If $B_{n}$ and $A_{n}$ are as in formulas (3.28) and (3.30), respectively, we can compute the modified approximants: $R_{n}(z, 1)=\frac{A_{n}(z, 1)}{B_{n}(z, 1)}$ and we obtain

$$
R_{n}(z, 1)=\frac{\left(1-z^{n}\right)(1-z)}{1-z^{n+1}}
$$

and the error for the Herglotz-Riesz transform is

$$
F_{\omega}(z)-R_{n}(z, 1)=\left\{\begin{array}{lll}
\frac{z^{n}(1-z)^{2}}{1-z^{n+1}} & \text { if } & |z|<1, \\
\frac{(1-z)^{2}}{z\left(1-z^{n+1}\right)} & \text { if } & |z|>1 .
\end{array}\right.
$$

Therefore, the error bound in this case is given by the following

Theorem 3.26. Let $f$ be analytic in $G=\{z: r<|z|<R, r<1, R>1\}$. Then for each $n$,

$$
\left|E_{n}(f)\right| \leq \frac{1}{2} \max _{\xi \in \Gamma}\left\{\left|\frac{f(\xi)}{\xi}\right|\right\}\left(\frac{r^{n+1}\left(1+r^{2}\right)}{1-r^{n+1}}+\frac{\left(1+R^{2}\right)}{R^{n+1}-1}\right),
$$

where $\Gamma=\Gamma_{1} \cup \Gamma_{2} . \Gamma_{1}$ and $\Gamma_{2}$ as in formula (3.13).

Finally, it should be indicated that a similar result to Remark 3.9 can be given for this case. 


\section{Numerical Results}

Let

$$
f_{1}(z)=\frac{\sin (z)}{4-z}, \quad f_{2}(z)=\frac{\sin \left(\frac{1}{z}\right)}{z-\frac{1}{4}}, \quad \text { and } \quad f_{3}(z)=\frac{\sin (z)}{(4-z)\left(z-\frac{1}{4}\right)} .
$$

Note that $\frac{f_{1}(z)}{z}$ is analytic for $0 \leq|z| \leq R$ when $R<4, \frac{f_{2}(z)}{z}$ is analytic for $r \leq|z| \leq \infty$ when $r>\frac{1}{4}$, and $\frac{f_{3}(z)}{z}$ is analytic for $r \leq|z| \leq R$ when $\frac{1}{4}<r<R<4$.

For these functions, we have calculated the exact error of the Szegö quadrature formula with respect to the weight functions $\omega(\theta)=\frac{\sin ^{2} \theta}{2 \pi}, \omega(\theta)=\frac{1+\cos \theta}{2 \pi}$ and $\omega(\theta)=\frac{1-\cos \theta}{2 \pi}$, respectively, and compared them with the error for the wellknown Gauss-Legendre quadrature formulas. Such quadrature formulas should be understood in the following sense (see [4, 5] $)$. Set $f\left(e^{i \theta}\right)=f_{1}(\theta)+i f_{2}(\theta)$. Thus,

$$
I_{\omega}(f)=\int_{0}^{2 \pi} F_{1}(\theta) d \theta+i \int_{0}^{2 \pi} F_{2}(\theta) d \theta
$$

where $F_{i}(\theta)=f_{i}(\theta) \omega(\theta), i=1,2$. If we estimate these latter integrals by means of the $n$-point Gauss-Legendre formula for $[0,2 \pi], \sum_{j=1}^{n} A_{j} F_{i}\left(\theta_{j}\right), i=1,2$ we can write

$$
I_{\omega}(f) \approx \sum_{j=1}^{n} A_{j}\left(F_{1}\left(\theta_{j}\right)+i F_{2}\left(\theta_{j}\right)\right)=\sum_{j=1}^{n} A_{j} f\left(e^{i \theta_{j}}\right) \omega\left(\theta_{j}\right)
$$

We also have calculated the error bounds given by formulas (3.14), (3.24) and (3.34). For the computation of Szegö quadrature formulas we have used Theorem 3.5, Theorem 3.14 and Theorem 3.23 for the weights functions $\omega(\theta)=\frac{\sin ^{2} \theta}{2 \pi}, \omega(\theta)=$ $\frac{1+\cos \theta}{2 \pi}$ and $\omega(\theta)=\frac{1-\cos \theta}{2 \pi}$, respectively.

For the weight function $\omega(\theta)=\frac{\sin ^{2} \theta}{2 \pi}$, we have the results appearing in Tables 13 , For the weight function $\omega(\theta)=\frac{1+\cos \theta}{2 \pi}$, we have the numerical results displayed in Tables 46. Finally, for the weight function $\omega(\theta)=\frac{1-\cos \theta}{2 \pi}$, we have the corresponding numerical results given in Tables 79

From the numerical results we see that Szegö formulas compete very favorably with Gauss-Legendre formulas. On the other hand, sharpness of the error bounds is also established in all of the examples.

TABle 1. $f_{1}(z)$

\begin{tabular}{|c|c|c|c|}
\hline Nodes & Exact error (Szegö) & Error bound $(R=3.8)$ & Exact error (Gauss-Legendre) \\
\hline$n=8$ & $1.24397935 \mathrm{E}-06$ & $2.431076 \mathrm{E}-05$ & $1.71024787 \mathrm{E}-03$ \\
$n=16$ & $9.68002240 \mathrm{E}-12$ & $5.591511 \mathrm{E}-10$ & $1.48264889 \mathrm{E}-07$ \\
$n=24$ & $1.54390389 \mathrm{E}-16$ & $1.286057 \mathrm{E}-14$ & $1.18979947 \mathrm{E}-11$ \\
\hline
\end{tabular}

TABle 2. $f_{2}(z)$

\begin{tabular}{|c|c|c|c|}
\hline Nodes & Exact error (Szegö) & Error bound $(r=0.27)$ & Exact error (Gauss-Legendre) \\
\hline$n=8$ & $1.990366 \mathrm{E}-05$ & $4.071163 \mathrm{E}-04$ & $3.156266 \mathrm{E}-02$ \\
$n=16$ & $1.548800 \mathrm{E}-10$ & $1.149814 \mathrm{E}-08$ & $4.977121 \mathrm{E}-06$ \\
$n=24$ & $2.178812 \mathrm{E}-15$ & $3.247415 \mathrm{E}-13$ & $7.953741 \mathrm{E}-08$ \\
\hline
\end{tabular}


TABle $3 . f_{3}(z)$

\begin{tabular}{|c|c|c|c|}
\hline Nodes & Exact error (Szegö) & Error bound $(R=3.8, r=0.27)$ & Exact error (Gauss-Legendre) \\
\hline$n=8$ & $7.400192 \mathrm{E}-07$ & $3.161199 \mathrm{E}-04$ & $3.116131 \mathrm{E}-04$ \\
$n=16$ & $1.091954 \mathrm{E}-11$ & $7.403863 \mathrm{E}-09$ & $2.943992 \mathrm{E}-10$ \\
$n=24$ & $2.775557 \mathrm{E}-17$ & $1.740482 \mathrm{E}-13$ & $4.424738 \mathrm{E}-09$ \\
\hline
\end{tabular}

TABle 4. $f_{1}(z)$

\begin{tabular}{|c|c|c|c|}
\hline Nodes & Exact error (Szegö) & Error bound $(R=3.8)$ & Exact error ( Gauss-Legendre) \\
\hline$n=8$ & $3.34996165 \mathrm{E}-06$ & $5.174076 \mathrm{E}-05$ & $3.50150187 \mathrm{E}-04$ \\
$n=16$ & $3.44166917 \mathrm{E}-11$ & $1.190039 \mathrm{E}-09$ & $1.43798826 \mathrm{E}-08$ \\
$n=24$ & $5.27355936 \mathrm{E}-16$ & $2.737112 \mathrm{E}-14$ & $1.26720897 \mathrm{E}-10$ \\
\hline
\end{tabular}

TABle $5 . f_{2}(z)$

\begin{tabular}{|c|c|c|c|}
\hline Nodes & Exact error (Szegö) & Error bound $(r=0.27)$ & Exact error (Gauss-Legendre) \\
\hline$n=8$ & $6.462231 \mathrm{E}-05$ & $8.689770 \mathrm{E}-04$ & $1.718254 \mathrm{E}-02$ \\
$n=16$ & $5.506783 \mathrm{E}-10$ & $2.454229 \mathrm{E}-08$ & $9.102588 \mathrm{E}-07$ \\
$n=24$ & $8.409939 \mathrm{E}-15$ & $6.931467 \mathrm{E}-13$ & $8.015859 \mathrm{E}-07$ \\
\hline
\end{tabular}

TABle $6 . f_{3}(z)$

\begin{tabular}{|c|c|c|c|}
\hline Nodes & Exact error (Szegö) & Eror bound $(R=3.8, r=0.27)$ & Exact error (Gauss-Legendre) \\
\hline$n=8$ & $2.614782 \mathrm{E}-06$ & $6.729565 \mathrm{E}-04$ & $1.895428 \mathrm{E}-04$ \\
$n=16$ & $3.882527 \mathrm{E}-11$ & $1.576203 \mathrm{E}-08$ & $1.192112 \mathrm{E}-07$ \\
$n=24$ & $4.996003 \mathrm{E}-16$ & $3.705510 \mathrm{E}-13$ & $9.867432 \mathrm{E}-08$ \\
\hline
\end{tabular}

TABle $7 . f_{1}(z)$

\begin{tabular}{|c|c|c|c|}
\hline Nodes & Exact error (Szegö) & Error bound $(R=3.8)$ & Exact error (Gauss-Legendre) \\
\hline$n=8$ & $2.08782660 \mathrm{E}-06$ & $5.174076 \mathrm{E}-05$ & $9.61621164 \mathrm{E}-04$ \\
$n=16$ & $1.23908591 \mathrm{E}-11$ & $1.190039 \mathrm{E}-09$ & $3.25547073 \mathrm{E}-08$ \\
$n=24$ & $1.90819582 \mathrm{E}-16$ & $2.737112 \mathrm{E}-14$ & $2.32242974 \mathrm{E}-11$ \\
\hline
\end{tabular}

TABle $8 . f_{2}(z)$

\begin{tabular}{|c|c|c|c|}
\hline Nodes & Exact error (Szegö) & Error bound $(r=0.27)$ & Exact error (Gauss-Legendre) \\
\hline$n=8$ & $2.238229 \mathrm{E}-05$ & $8.689770 \mathrm{E}-04$ & $6.482502 \mathrm{E}-03$ \\
$n=16$ & $1.982434 \mathrm{E}-10$ & $2.454229 \mathrm{E}-08$ & $8.908710 \mathrm{E}-07$ \\
$n=24$ & $2.955968 \mathrm{E}-15$ & $6.931467 \mathrm{E}-13$ & $5.565306 \mathrm{E}-08$ \\
\hline
\end{tabular}

TABle $9 . f_{3}(z)$

\begin{tabular}{|c|c|c|c|}
\hline Nodes & Exact error (Szegö) & Error bound $(R=3.8, r=0.27)$ & Exact error (Gauss-Legendre) \\
\hline$n=8$ & $9.413287 \mathrm{E}-07$ & $6.729565 \mathrm{E}-04$ & $1.330823 \mathrm{E}-04$ \\
$n=16$ & $1.397712 \mathrm{E}-11$ & $1.576203 \mathrm{E}-08$ & $2.980441 \mathrm{E}-08$ \\
$n=24$ & $2.220446 \mathrm{E}-16$ & $3.705510 \mathrm{E}-13$ & $7.450711 \mathrm{E}-08$ \\
\hline
\end{tabular}




\section{REFERENCES}

[1] N. I. Akhiezer. The classical moment problem and some related questions in analysis. Hafner, New York (1965) MR 32:1518

[2] A. Bultheel. Algorithms to compute the reflection coefficients of digital filters. Numerical Methods of Approximation Theory, Vol. 7 eds. L. Collatz, G. Meinardus and H. Werner (Birkhåuser, Basel, 1984) pp. 33-50. MR 85m:94011

[3] L. Daruis and P. González-Vera. Szegö polynomials and quadrature formulas on the unit circle. Applied Numerical Mathematics 36 (2001) 79-112. CMP 2001:07

[4] W. Gautschi and G. V. Milovanović. Polynomials orthogonal on the semicircle. Journal of Approximation Theory 46 (3) (1986) 230-250. MR 87g:33005

[5] P. González-Vera, J. C. Santos-León and O. Njåstad. Some results about numerical quadrature on the unit circle. Advances in Computational Mathematics 5(1996) pp. 297-328. MR 98f: 41028

[6] W.B. Jones, O. Njåstad and W. J. Thron. Moment theory, orthogonal polynomials, quadrature, and continued fractions associated with the unit circle. Bull. London Math. Soc. 21 (1989) pp. 113-152. MR 90e:42027

[7] W.B. Jones, H. Waadeland. Bounds for remainder terms in Szegö quadrature on the unit circle. Applied Numerical Mathematics. International Series of Numerical Mathematics. Vol. 119, (1994) Birkhäuser. 325-342. MR 96i:41029

[8] A. Magnus. Semiclassical orthogonal polynomials on the unit circle. MAPA 3072. Special topics in Approximation Theory. Unpublished Technical Report. (1999)

[9] F. Peherstorfer. A Special Class of Polynomials Orthogonal on the Unit Circle including the Associated Polynomials. Constr. Approx. 12 (1996) pp. 161-185. MR 97d:42023

[10] F. Peherstorfer, R. Steinbauer. Characterization of Orthogonal Polynomials with respect to a Functional. Journal of Computational and Applied Mathematics 65 (1995) pp. 339-355. MR 97b:42045

[11] G. Szegö. Orthogonal Polynomials. American Mathematical Society, 4 th. ed., Vol. 23, (Colloquium Publications), 1975. MR 51:8724

Department of Mathematical Analysis, La Laguna University, Tenerife, Canary IsLANDS, SPAIN

E-mail address: ldaruis@ull.es

Corresponding author: Department of Mathematical Analysis, La Laguna UniverSity, 38271- La Laguna, Tenerife, Spain

E-mail address: pglez@ull.es. Fax: 34-922-318195

Department of Mathematical Sciences, Norwegian University of Science and TechNOLOGY, Trondheim, NorWAY

E-mail address: njastad@math.ntnu.no 